\title{
mmWave mobile communication under hypercellular architecture
}

\author{
LI Jiahui, HUANG Fei, ZHOU Runyun, CHEN Wenhua, TIAN Zhigang, ZHOU Shidong
}

Department of Electronic Engineering, Tsinghua University, Beijing 100084, China

\begin{abstract}
To meet the ever growing traffic in mobile communication, mmWave (millimeter-Wave) frequency bands have gained considerable attention for having a greater amount of bandwidth available than the current cellular spectrum below $3 \mathrm{GHz}$. Several test systems have been reported on recently to validate the possibility of mmWave links in mobile scenarios. However, there still exist practical issues to enable the application of mmWave in mobile communication, including reliability and cost. In this article, we present some new designs that address these issues, where system architecture, transceiver architecture, and related issues such as circuits and antenna arrays are considered. Hypercellular architecture is applied in mmWave mobile networks to overcome blockage problems, and a Butler-matrix-based HBF (Hybrid Beamforming) architecture is considered in an mmWave link. Simulations and experimental results are presented to validate the effectiveness of the Butler-matrix-based system.
\end{abstract}

Key words: Butler matrix, hypercellular architecture, hybrid beamforming, millimeter wave, transceiver architecture

Citation: LI J H, HUANG F, ZHOU R Y, et al. mmWave mobile communication under hypercellular architecture[J]. Journal of communications and information networks, 2016, 1(2): 62-76.

\section{Introduction}

In recent years, the demand for cellular data has been growing at an explosive rate. Mobile data traffic has grown 4000 -fold over the past 10 years and almost 400 -million-fold over the past 15 years $^{[1]}$. Global mobile traffic reached 3.7 exabytes per month at the end of 2015, up from 2.1 exabytes per month at the end of $2014^{[1]}$. However, the bandwidth available in the current cellular spectrum below $6 \mathrm{GHz}$ is too limited. In order to meet future demands on capacity and data rates, extending the current cellular spectrum to mmWave bands (around $30 \mathrm{GHz}$ and beyond) has gained considerable attention ${ }^{[2-4]}$.

As proven by measurement campaigns shown in Ref.[5], mmWave (millimeter-Wave) signals suffer from severe path loss, penetration loss, rain effects, and atmospheric absorption. Therefore, a large number of antennas should be configured at the BS (Base Station) in an mmWave communication system

Manuscript received Jun.16, 2016; accepted Aug.18, 2016

This work was supported by The National Basic Research Program of China (No.2012CB316002), The National Natural Science Foundation of China (No.61201192), The National High Technology Research and Development Program of China (863 Program) (No.2014AA01A703), The Science Fund for Creative Research Groups of NSFC (No.61321061), The International Science and Technology Cooperation Program (No.2012DFG12010), The National S\&T Major Project (No.2016ZX03001005), The Key Grant Project of Chinese Ministry of Education (No.313005), The Tsinghua University Initiative Scientific Research (No.2015Z02-3), The Open Research Fund of National Mobile Communications Research Laboratory, Southeast University (No.2012D02), The Tsinghua-Qualcomm Joint Research Program, and the Intel Collaborative Research Institute for Mobile Networking and Computing. 
to provide agile beamforming and compensate for the severe signal attenuation.

Several test systems have been established to prove the possibility of mobile coverage using mmWave. Samsung developed a 28-GHz prototype and tested its peak data rate and mobility properties in an outdoor cellular scenario ${ }^{[6]}$. In addition, NTT DOCOMO developed a 73.5-GHz experimental system and tested the signal gains of all possible beam directions achieved with the system ${ }^{[7]}$. These results imply that an mmWave communication system can perform well under such test scenarios, at least in some locations.

However, mobile mmWave links based on beamforming with high directional gain may experience a high probability of blockage. This is because the number of mmWave propagation paths with enough strength is very limited, and the abrupt loss of tens of $\mathrm{dB}$ may occur in milliseconds ${ }^{[8]}$, resulting in frequent loss of control for link adaptation. Thus, an mmWave communication system is more sensitive to blockage ${ }^{[3,4]}$, and there exist challenges in providing mmWave with effective coverage in highmobility and high-reliability scenarios.

Hypercellular architecture, where the coverage of control and traffic data can be designed and deployed separately ${ }^{[9]}$, provides a way to solve these difficulties by providing seamless coverage of control.

In this paper, we present some new designs that consider implementation issues and analyze system performance, where network architecture, transceiver architecture, and related issues such as circuits and antenna arrays are all considered. For network architecture, a hypercellular architecture with mmWave is proposed, in which the mmWave band is utilized for traffic data coverage with control coverage provided by a low-frequency band. For transceiver architecture, beamforming is necessary to provide enough signal power gain ${ }^{[5]}$. Owing to the fact that $\mathrm{ABF}$ (Analog Beamforming) is very important in an mmWave system ${ }^{[10]}$, a possible solution that utilizes the Butler matrix phase-shifting network is proposed.

The rest of the article is organized as follows. The mmWave network hypercellular architecture and transceiver architecture are discussed in Sections 2 and 3, respectively. Then, the application of a Butler matrix to mmWave mobile communication is presented in Section 4. In Section 5, the channel estimation and beamforming algorithms for the Butler-matrix-based system are provided, and the system performance using such algorithms is evaluated in Section 6. Moreover, the test bench for the Butler-matrix-based system is presented with some experimental results in Section 7. Our conclusions are drawn in Section 8.

\section{Hypercellular network architecture with mmWave}

The architecture of an mmWave hypercellular network with a separation of the control plane and data plane for $5 \mathrm{G}$ (Fifth-Generation communication) is shown in Fig.1. Two different coverages are provided for each user, namely, traffic coverage and control coverage. Under the separation of the control plane and traffic plane, mmWave BSs performing as microcells, or traffic BSs, will be deployed densely, providing an extremely high traffic data rate. In addition, another layer of coverage is provided by the control BS, providing seamless reliable mobile coverage for crucial control signaling, using the existing mobile spectrum below $3 \mathrm{GHz}$. These control BSs can be realized by using newly deployed BSs and by upgrading existing $4 \mathrm{G}$ BSs. In addition to reducing control overhead ${ }^{[4,9,11]}$, the mmWave hypercellular architecture with separated coverage for signaling and traffic has the following additional advantages:

Providing smooth and efficient handoff for user mobility. Owing to the limited output power of mmWave RF and the high propagation loss, the 
range of an mmWave cell will be relatively smaller than that operating in a lower frequency band. As a result, a moving piece of UE (User Equipment) may experience frequent handoffs between different mmWave BSs. In addition, since mmWave mobile transmission relies on propagation through very few paths, which may experience abrupt blockage, the connection can deteriorate too quickly to enable a handoff operation. In a hypercellular architecture, the handoff operation can be handled in signaling coverage with much higher reliability, which can support the smooth handoff of traffic coverage (between cells and between beams) in the mmWave band.

Capability of optimizing the coverage of control data and traffic data separately. As we mentioned previously, a narrow beam is essential in mmWave bands to support a high data rate, but some signaling (especially for those in access control and handoff control) needs to be broadcast. A flexible hypercellular architecture is very suitable to support separately designed coverage and streams for signaling data and traffic data.

\section{$3 \mathbf{m m W a v e}$ transceiver architecture}

Full digital beamforming, as shown in Fig.2, is a straightforward method using MIMO (MultiInput Multi-Output) techniques, which can provide full flexibility in design and can achieve the best performance in terms of spectrum efficiency. However, the huge amount of RF chains and $\mathrm{AD} / \mathrm{DA}$ (Analog-to-Digital/Digital-to-Analog) devices may require significant costs and power consumption, which may not be suitable for commercial use $\mathrm{e}^{[12]}$. In order to handle these problems, HBF is proposed to reduce the number of RF chains and to provide good performance ${ }^{[10]}$. An HBF consists of an ABF stage and a DBF (Digital Beamforming) stage. For example, in downlink transmission, the basic idea of HBF at the BS side is to first process the transmitted signals in the digital domain with a relatively low dimension, and then feed this to the large number of antenna elements through an analog phase-shifting network.

Consider the realization of an HBF system. As shown in Fig. 3 and Fig.4, the PAs can be placed

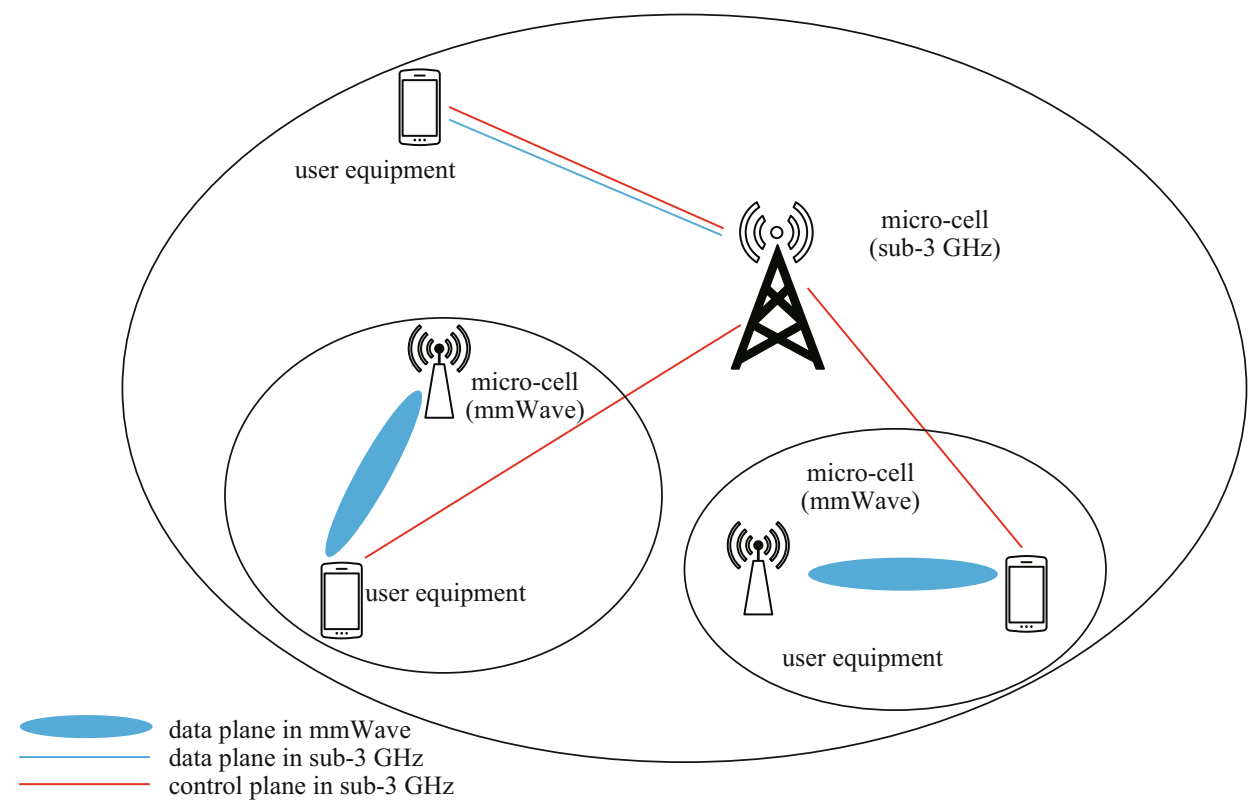

Figure 1 Architecture of mmWave mobile network 


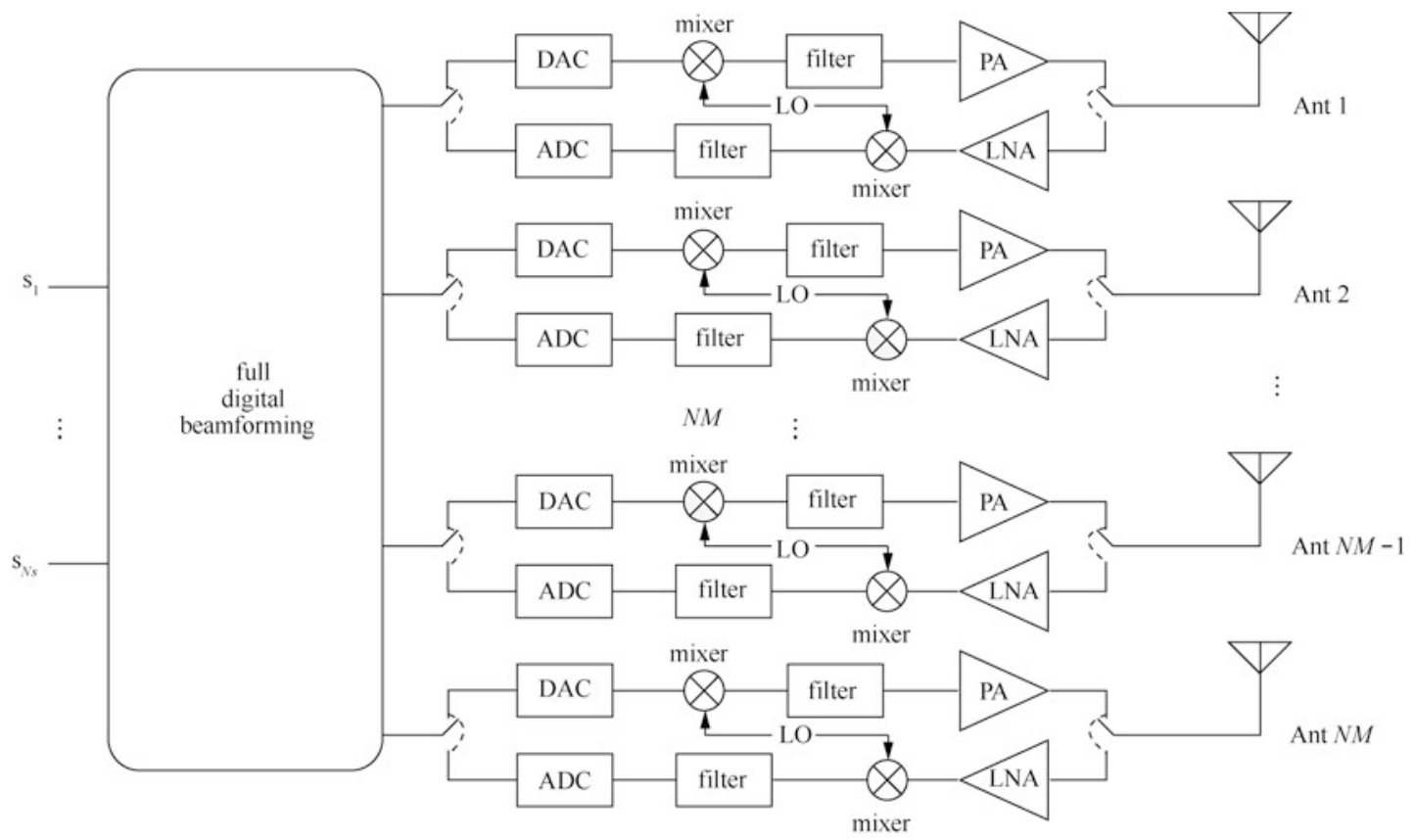

Figure 2 Full digital beamforming system structure

in different places. When placing the PAs in the RF chains, the number of PAs needed is much smaller than a structure where they are placed near the antennas. However, the power requirement for each PA is higher, owing to the fact that each PA should amplify the signal corresponding to multiple antennas. In addition, the power loss in the ABF stage can reduce the total power requirement when the PAs are placed near the antennas and the gain of each PA is fixed. Thus, placing the PAs near the antennas is a better choice for cases in which the loss introduced by the phase-shifting network is

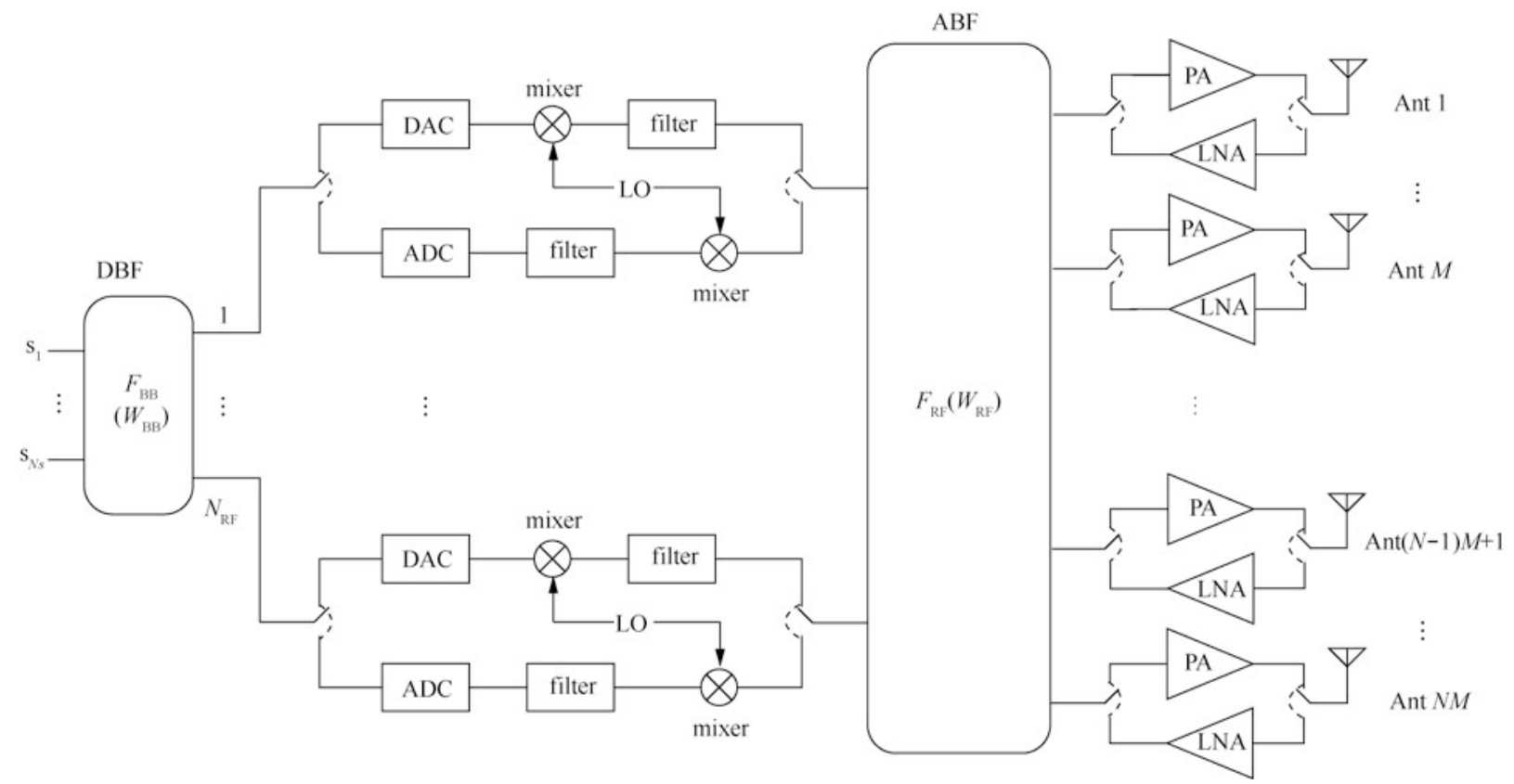

Figure 3 Hybrid beamforming structure with PAs near the antennas 


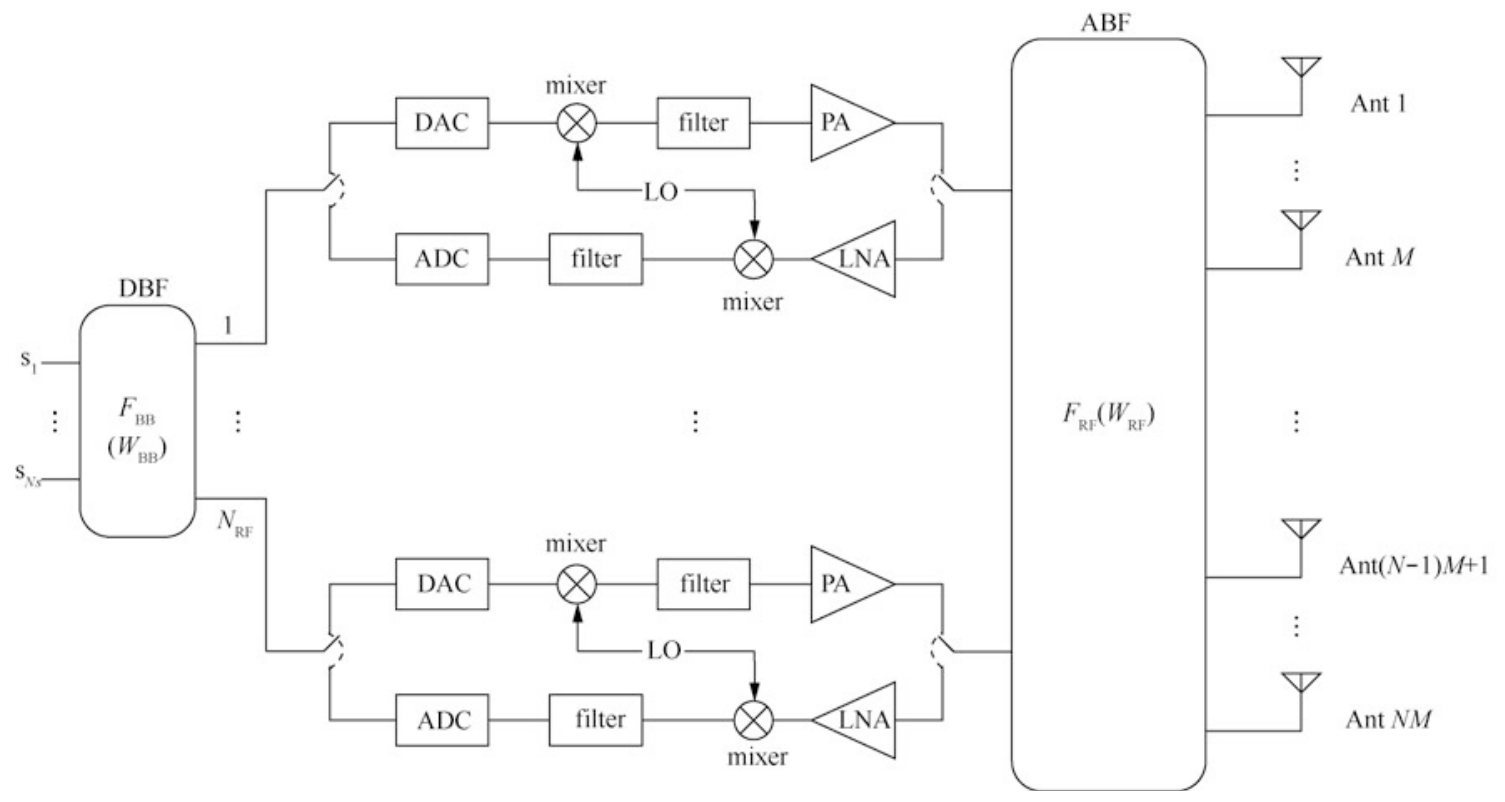

Figure 4 Hybrid beamforming structure with PAs in the RFs

very large.

In Fig.5, we can see that the ABF stage can be achieved with two different structures: a subconnected structure and a fully connected structure. In the subconnected structure, each RF is connected to the antennas in an independent subarray. In the fully connected structure, each RF is connected to each antenna. It is obvious that the latter arrangement can (a)

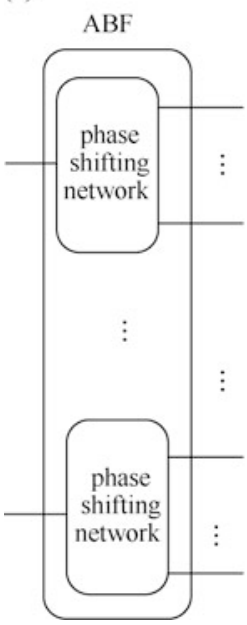

(b)

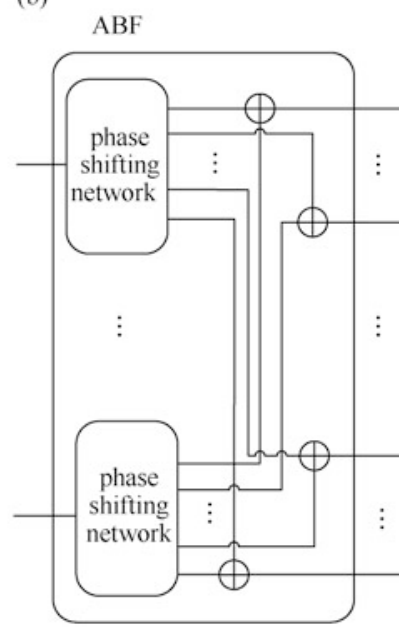

Figure 5 Analog beamforming structure: (a) subconnected structure; (b) fully connected structure achieve a better rate of performance, owing to the fact that it can realize more possible beams. However, a much more complicated circuit is needed in the latter structure, which makes it difficult to implement in a real system.

There are at least two kinds of structures that can realize the $\mathrm{ABF}$ stage, i.e., an $\mathrm{ABF}$ with variable phase shifters (called the phase-shifter-based structure) ${ }^{[10]}$, and fixed passive beamformers with RF switches.

Variable phase shifters can be realized as either active phase shifters or passive phase shifters. For the active phase shifters, power consumption is high, and nonlinearity problems will result in a high noise figure $^{[13]}$. For the passive variable phase shifters, different phase shifting values result in different insertion loss, which will cause a negative impact on system performance. It is difficult to compensate for this effect under passive conditions ${ }^{[13]}$.

RF switches for fixed passive beamformers may have different types of implementations, for example, a microwave-lens-based structure ${ }^{[14]}$ or a Butlermatrix-based structure ${ }^{[15]}$.

This kind of structure has the following characteristics:

1) No active component in the signal path; 
2) Low power consumption and low insertion loss;

3) Different beams of fixed directional pattern;

4) Different beams sharing the same antenna aperture;

5) RF switches are used for beam selection;

6) No need for a power amplifier at each antenna element, avoiding real-time calibration of the beams.

This is realized by using a Butler phase shifting matrix, which consists of a fixed-phase shifting network that can achieve several fixed beam directions, which is equivalent to a predefined codebook. An RF switch is configured to choose the beam direction of each Butler matrix.

\section{Butler-matrix-based front end}

As mentioned before, various types of beam patterns can be generated based on the multiple antenna structure of the system, wherein the switched-beam pattern is probably the most commonly used owing to its simple implementation and easy-to-control mechanism. Traditional phased-array antennas utilize phase shifters integrated on the substrate for controlled beam steering. However, there are limited types of low-loss phase shifters available for small-sized antenna applications at millimeter-wave frequencies ${ }^{[16,17]}$. The inclusion of several integrated circuits or multilayered structures necessary to achieve a wide beam scan drives up the costs. A combined solution to these requirements is the integration of a switched beamforming network that implements a phased-array scanning capability. One such structure is the Butler matrix, which consists of $\mathrm{N}$ number of RF inputs that independently feed $N$ number of RF outputs with different phase delays ${ }^{[18]}$. This creates a number of fixed radiating beams that are accessed by the separate inputs.

For the millimeter wave, additional different main direction beams are required to build the communication link. The traditional distributed phased array needs a phase shifter for each antenna and each beam. In order to realize a feeding network with $N$ beams (a fully connected ABF structure), $N^{2}$ phase shifters are required to steer the phases of signals. Therefore, the straightforward phased array is very costly and unsustainable when the beamforming gain increases (increasing $N$ ).

\subsection{Butler matrix}

The Butler matrix is a passive beamforming circuit that can generate $N$ phase distributions with equal amplitudes through different feeding ports ${ }^{[19,20]}$. It consists of $90^{\circ}$ hybrid couplers, crossovers, and several kinds of phase shifters. The number $N(N=$ $2^{K}$ ) of radiating beams created by a Butler matrix is equal to the number of antennas in the array that is fed by Butler matrix network. In order to implement an $N$-way Butler matrix network, $N_{\mathrm{C}}$ hybrid couplers and $N_{\mathrm{P}}$ phase shifters are used ${ }^{[20]}$. An antenna array excited by $N$ output signals with equal power levels and progressive phases creates $N$ kinds of different main direction beams.

$$
\begin{gathered}
N_{\mathrm{C}}=\frac{N}{2} \mathrm{lb} N=\frac{N K}{2}, \\
N_{\mathrm{P}}=\frac{N}{2}(\mathrm{lb} N-1)=N_{\mathrm{C}}-\frac{N}{2} .
\end{gathered}
$$

Thus, it is seen that a Butler matrix has a much simpler implementation, lower transmission loss, and lower cost, and is scalable to very large arrays, compared with a traditional distributed phased feeding network. Hence, the Butler matrix is suitable to realize a switched-beam antenna array in millimeter-wave wireless communication.

\subsection{Energy-efficient PA design}

In the structure of an $\mathrm{ABF}$ with fixed passive beamformers, since the insertion loss is quite small, a power amplifier can be allocated in the RF chain to 
reduce the required number of PAs, and to avoid realtime calibration of the beams. However, these benefits depend on energy-efficient PA designs.

Owing to the currently limited efficiency in the mmWave band, a high-efficiency PA will be a key component in the beamforming-based array transmitter. As shown in Fig.6 and Fig.7, ET (Envelope-Tracking) and Doherty architectures are very promising solutions for the BSs and the UEs, respectively $^{[21]}$. For 5G applications, the instantaneous bandwidth will be wider than $500 \mathrm{MHz}$, which is impractical for ET architecture owing to the bandwidth bottleneck of the envelope amplifier. Therefore, it can be estimated that the Doherty architecture will continue to dominate in emerging wireless communication systems.

Since the output of each PA device in a massive MIMO architecture is trivial, it could be less than $30 \mathrm{dBm}$ owing to the output limitation of semiconductor devices in the mmWave band, and

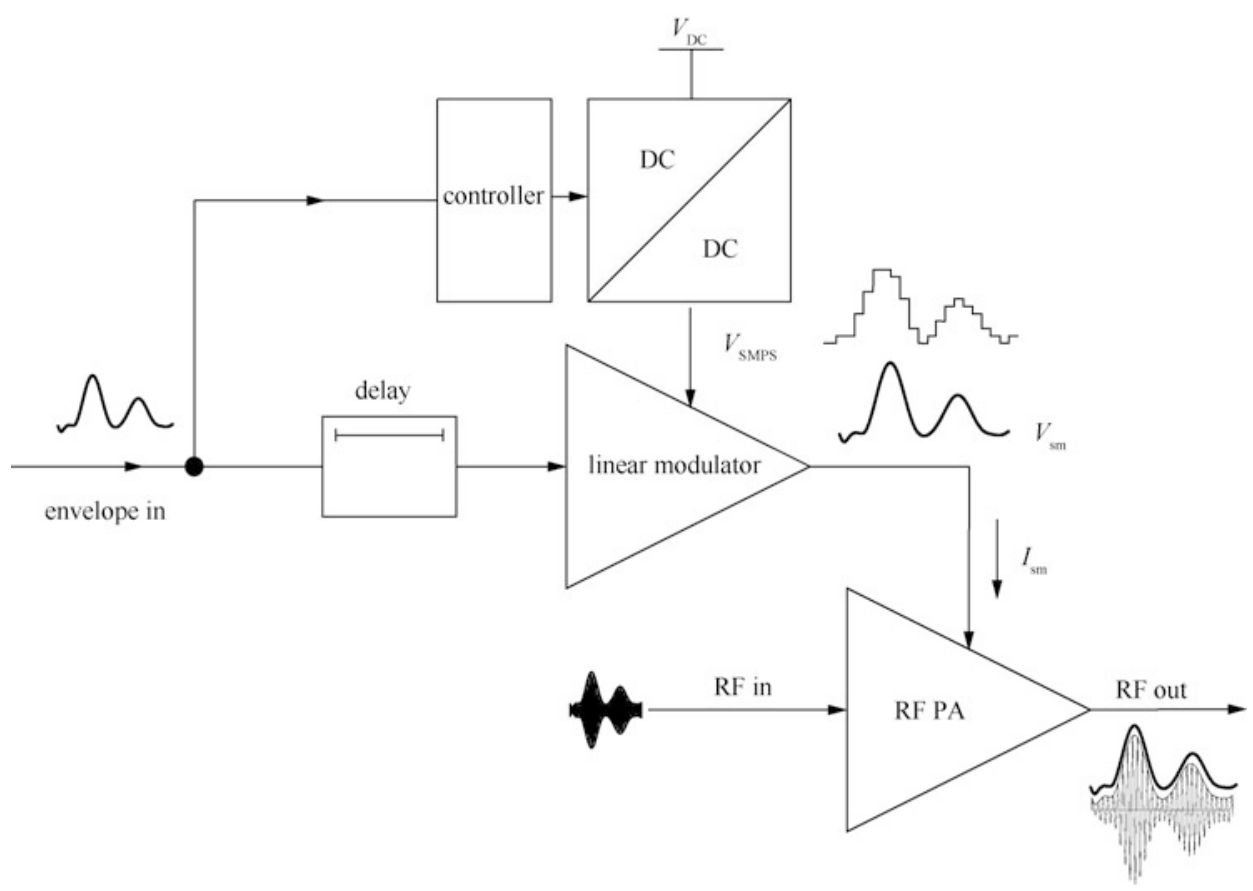

Figure 6 Energy-efficient PA architectures for 5G: envelope tracking

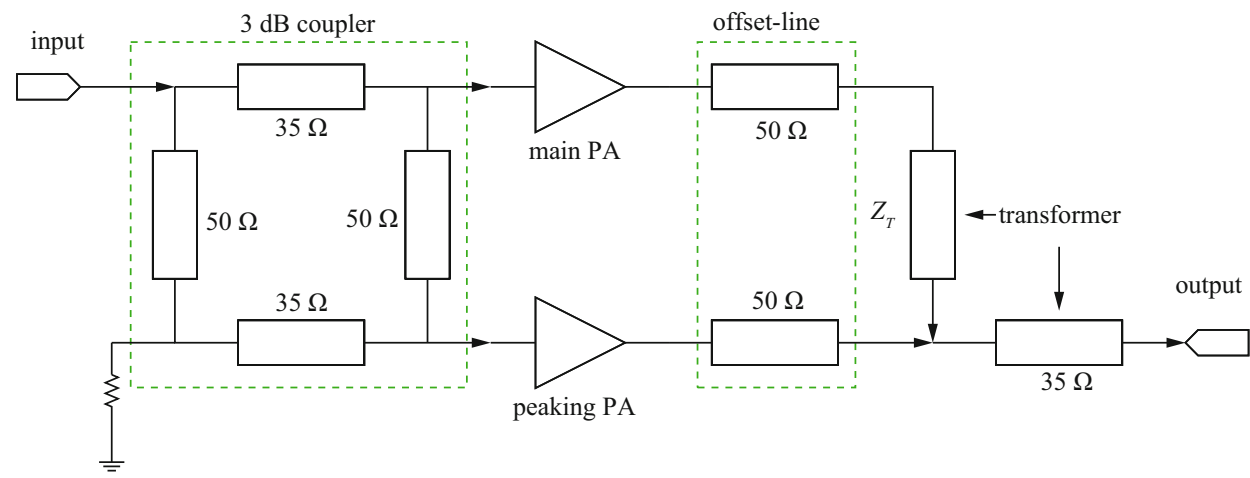

Figure 7 Energy-efficient PA architectures for 5G: Doherty 
the array gain of the beamforming transmitter. The DPD (Digital Predistortion) technique will be precluded owing to the significant power consumption of the DPD process module and broadband feedback loop. In this situation, novel linearization techniques should be adopted for mmWave Doherty PA design. For example, the nonlinear intermodulation canceling method can be employed for PA linearization.

\section{Hybrid beamforming algorithms}

As mentioned previously, mmWave transmission depends on very few propagation paths with very small angular spread; therefore, effective beamforming algorithms are needed to cope with time-varying propagation parameters.

Actually, different algorithms are needed for different transceiver architectures. In this paper, we show an example of the transceiver architecture using a fixed passive beamformer, such as the Butlermatrix-based HBF structure. Here, the algorithms of the variable-phase-shifter-based structure cannot be directly utilized for fixed beams. Thus, we need to consider how to perform related channel estimation and beamforming under such constraints.

\subsection{Channel estimation}

In a Butler-matrix-based system, we have only a limited number of analog beam directions to choose from, which should be fully utilized in both the channel estimation stage and the HBF stage. To acquire CSI (Channel State Information) at the BS and from each user, there are two main possible methods.

The first method is to conduct beam sweeping to obtain the best analog beam directions corresponding to each RF chain. Then, channel estimation is done to the effective channel with the chosen analog beams.
The effective channel is defined as the channel between different RF chains using different analog beams. This means that for each combination of transmit-and-receive RF chains, each combination of analog beams corresponds to an independent effective channel coefficient.

The second method is to measure the channel using all possible beams. Thus, we can obtain the equivalent channel response matrix between each RF chain at the BS and each RF chain at each user, when using different analog transmit and receive beams. Since the beamforming vectors in each codebook can form an invertible matrix, the channel matrix between transmit and receive antennas can be estimated by using a least-square scheme.

\subsection{Hybrid beamforming}

When utilizing the first method of channel estimation, the ABF matrix can be obtained by choosing the beam combinations that have the largest effective gain with the estimated effective channel. The DBF matrix is calculated by using a BD (Block Diagonalization) algorithm ${ }^{[22]}$ with the estimated effective channel.

For the second method of channel estimation, we can determine the estimated channel matrix between the transmit and receive antennas. The $\mathrm{ABF}$ and $\mathrm{DBF}$ matrices are calculated with the estimated channel. During the ABF stage, our goal is to find the analog beams that maximize the capacity of the effective channel, which considers the effect of ABF. Since there are a limited number of analog beams to be chosen, an exhaustive search can be utilized to find the best combination of beams. This is described in detail in Ref.[23]. It can achieve a better performance than the first method because it considers the maximization of capacity, while the first method only considers how to maximize the effective gains. Then, in the DBF stage, we can use the $\mathrm{BD}$ algorithm with the low-dimensional effective channel to get the DBF matrix. 


\section{System performance evaluation}

In this section, the performance of the Butler-matrixbased HBF system with the algorithms introduced in Section 5 is analyzed, including the SE (Spectral Efficiency) and EE (Energy Efficiency). Here, we consider the much more realistic subconnected structure and utilize the second method of channel estimation. The subconnected full digital system and the phase-shifter-based HBF system with algorithms given by Ref.[24] are utilized for comparison. We assume transmission over flat-fading channels. To model the mmWave propagation environment, a clustered multipath channel model is utilized ${ }^{[25]}$.

The SE is obtained by calculating the ergodic achievable downlink sum rate under different SNRs (Signal-to-Noise Ratios). Using the power consumption model introduced in Ref.[13], we calculate the total power consumption of the system and obtain EE with $E E=W \times S E / P_{\text {total }}$, where $W$ is the bandwidth of the system, and $P_{\text {total }}$ is the total power consumption.

One example is shown here to evaluate the performance of the Butler-matrix-based HBF system, where the BS and UEs all use the subconnected Butler-matrixbased structure. Consider the downlink of a singlecell multiuser mmWave system with ULAs that have half-wavelength antenna spacing. The BS is equipped with 32 transmit antennas and four RF chains. Each RF chain is connected to a subarray with eight antennas through a Butler matrix. There are two users served simultaneously by the BS. Each user has eight antennas and two RF chains. Each RF chain of users is connected to a subarray with four antennas through a Butler matrix. The transmitting data stream number is assumed to be equal to the number of RF chains. The channels are generated according to the clustered multipath channel model with six scattering clusters, each having five propagation paths. The cluster power is randomly generated from a uniform random variable distributed in $[0,1]$, and then the total cluster power corresponding to each user is normalized. The azimuth AoA (Angle of Arrival) and AoD (Angle of Departure) within a cluster are assumed to be Laplacian distributed with an angular standard deviation of $5^{\text {o[25] }}$. The mean cluster angles are assumed to be uniformly distributed within [0, $360^{\circ}$ ]. The codebooks consist of beamforming vectors are chosen from the DFT matrices. Therefore, the sizes of the codebooks for the BS and users are eight and four, respectively.

The ergodic performance of achievable downlink sum SE and the SNR is shown in Fig.8. It can be seen that the Butler-matrix-based system can achieve $90 \%$ of the sum achieved by SE by the variable-phaseshifter-based system, and $85 \%$ of that achieved by the full digital system.

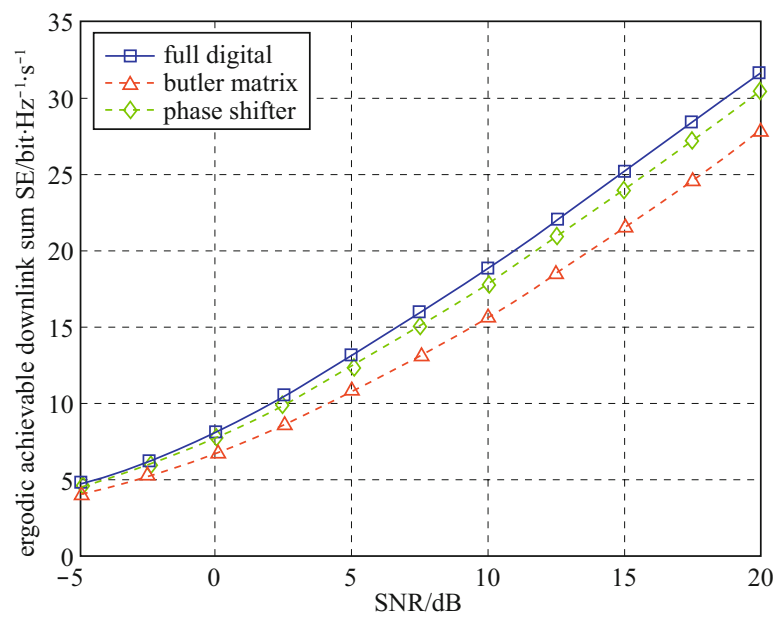

Figure 8 Ergodic achievable downlink sum SE and SNR

Assume that the average path loss is $120 \mathrm{~dB}$, and each antenna has a gain of $10 \mathrm{~dB}$. The noise power spectral density is $-174 \mathrm{dBm} / \mathrm{Hz}$, and the bandwidth of the system is set as $200 \mathrm{MHz}$. Each time we are given an SNR, we can calculate the average transmitting power of an antenna at the BS side and get the power consumption of the BS. Then, the EE can be obtained. 
The tradeoff of EE vs. SE for different HBF schemes is shown in Fig.9. It can be seen that as SE increases, the changing trend of EE is similar for all systems. When SE is low, EE increases with SE. After reaching a peak, EE decreases with SE. The peak is called the green point ${ }^{[26]}$. It is obvious that the HBF system performs with more energy efficiency than the full digital system when SE is lower than $21 \mathrm{bit} \cdot \mathrm{s}^{-1} \cdot \mathrm{Hz}^{-1}$. Considering HBF systems, we can see that the Butler-matrix-based system achieves a better EE performance than the phase-shifter-based system. The green point obtained by the Butler-matrix-based system has an improvement of $27 \%$ compared with the phase-shifter-based system. When SE is high, we can see that the Butler-matrix-based system performs worse than the phase-shifter-based system. This is because a high SNR is needed to support the high SE, which results in a high transmit power. Therefore, the total power consumption is mainly caused by Pas. Thus, when the SNR is the same, both HBF systems have similar total power consumption, and the power saved by the Butler matrix is trivial. Since the phaseshifter-based system has a better SE performance when the SNR is the same, it is reasonable that the

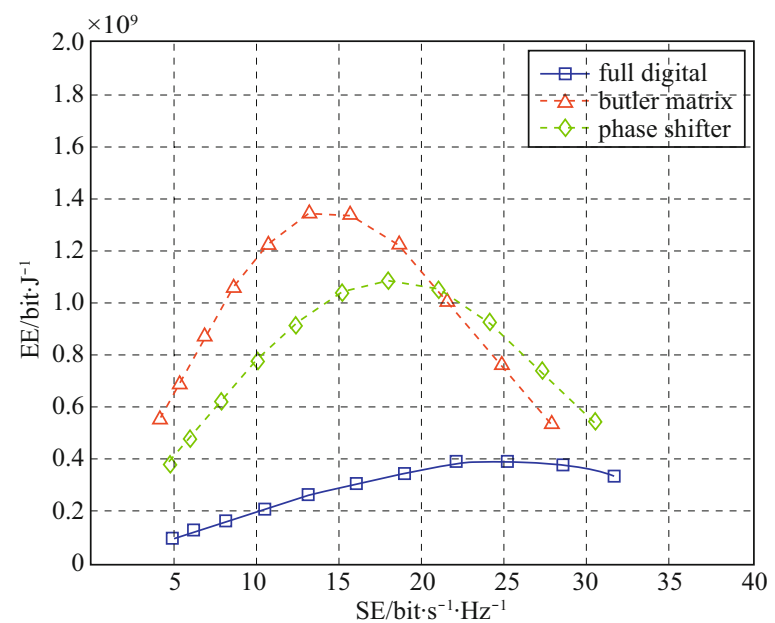

Figure 9 EE and SE. for different HBF schemes

Butler-matrix-based system performs worse than the phase-shifter-based system when SE is high.

Therefore, it can be concluded that the Butlermatrix-based system can achieve an acceptable SE performance compared with the phase-shifter-based system, and a better EE performance can be obtained.

\section{Test bench design and testing}

In this section, the test bench of the hypercellular architecture with mmWave is introduced. Fig.10

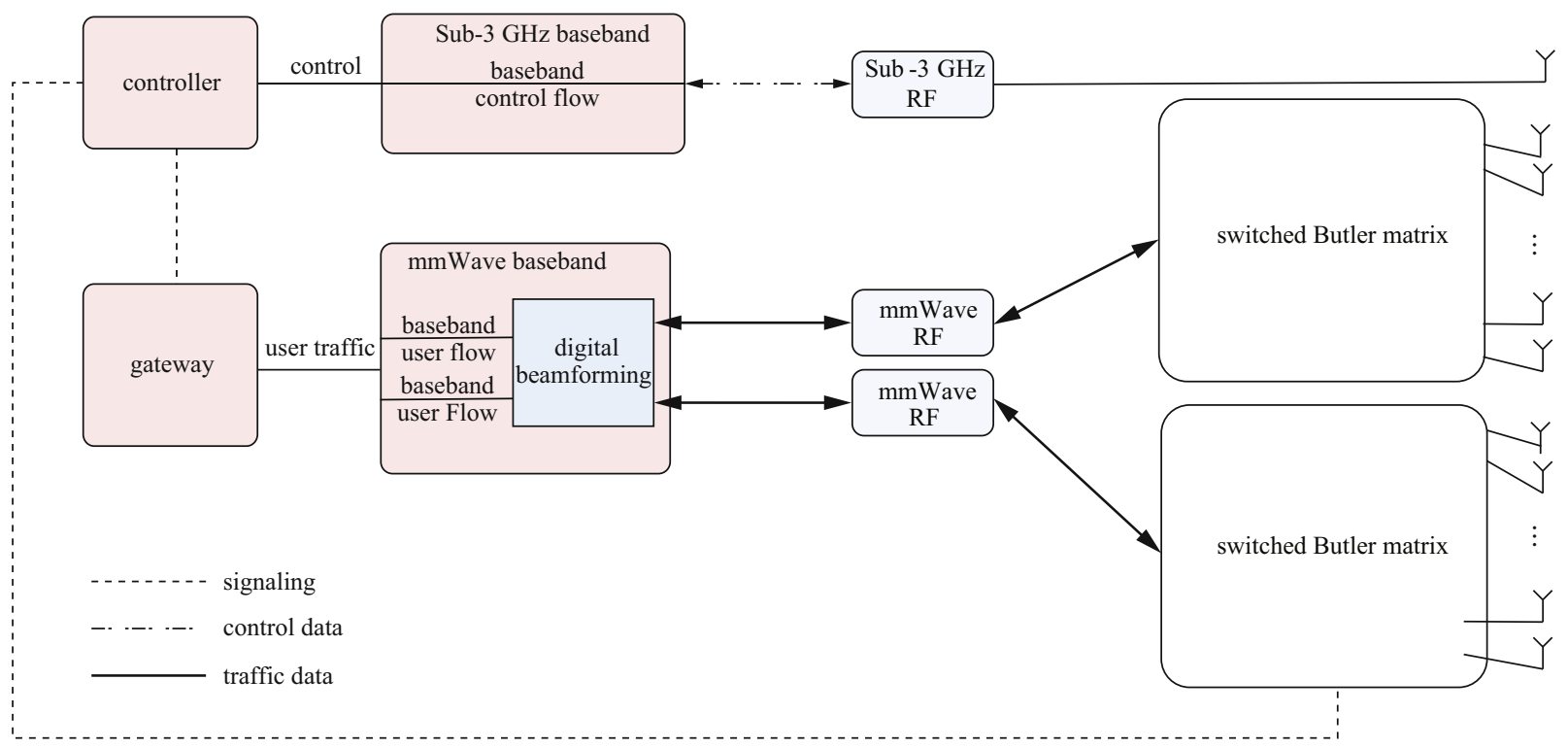

Figure 10 Architecture of test bench 
shows the design architecture at the network side on the test bench, including an mmWave traffic BS and a control BS in the lower spectrum. The architecture of the UE is symmetrical to the BS. In the system, $25.6 \mathrm{GHz}$ is used to transmit downlink data. A hybrid beamforming architecture with a subconnected Butler-matrix-based ABF is applied, while the control coverage is implemented with an LTE (Long Term Evolution) frequency.

There are two baseband processing programs running in the BS server. One of them is the baseband of an mmWave BS, which is responsible for traffic data. Another is responsible for control data and assists the mmWave BS in completing beam sweeping and tracking.

\subsection{Design and testing of Butler matrix}

Fig. 11 shows the design of the switched-beam patch antenna array. The array is formed by an antenna array and the Butler matrix, which works as the feeding network and switch network.

The purpose of this network is to uniformly feed the eight antenna elements with progressive phase delays of $+22.5^{\circ},-22.5^{\circ},+67.5^{\circ},-67.5^{\circ},+112.5^{\circ}$, $-112.5^{\circ},+157.5^{\circ}$, and $-157.5^{\circ}$, which are determined by the respective input port selected.

In order to combine the antenna array and the

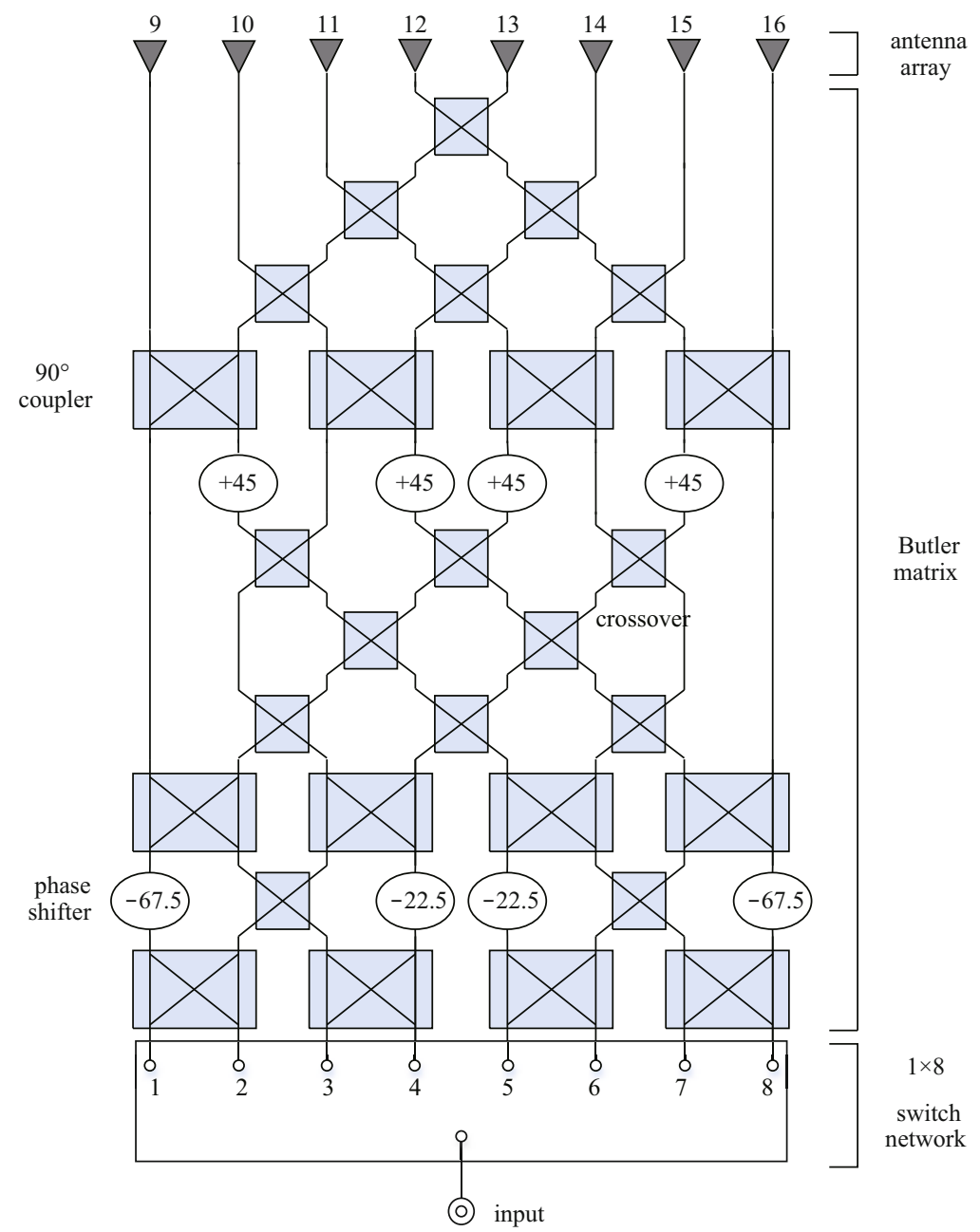

Figure 11 Design of switched-beam patch antenna array based on Butler matrix 
switch network, the Butler matrix network is basically implemented with $50-\Omega$ microstrip lines. To prevent the propagation of substrate and surface waves, which affect the antenna characteristics, a Taconic RF35tc substrate with a dielectric constant of $\varepsilon_{r}=3.5$ and a thickness of 5 mil was chosen.

A $90^{\circ}$ hybrid coupler that can generate signals $90^{\circ}$ out of phase at its outputs consists of two pairs of $50-\Omega$ and $35.5-\Omega$ quarter-wavelength microstrip lines. The use of hybrid couplers provides high isolation between each input port, which allows a switch network to toggle between these ports without affecting the antenna performance. Crossover that can cross two transmission lines without a loss of power is implemented by cascading two $90^{\circ}$ hybrid couplers. The phase shifter uses phase-delay lines to obtain a change of phase as $-22.5^{\circ}$ or $-67.5^{\circ}$. The antenna array is implemented in a Taconic RF35 substrate with a dielectric constant of $\varepsilon_{r}=3.5$ and a thickness of 20 mil because of its wide bandwidth. Owing to the accurate beam pointing, the distance between the centers of two adjacent patches is $6 \mathrm{~mm}$, and $\lambda_{0} / 2$ at $25 \mathrm{GHz}$.

The fabricated prototype is shown in Fig.12. The size of the entire microstrip circuit is approximately $150 \times 126 \mathrm{~mm}^{2}$. The simulated phase differences between two adjacent output ports are $-26.2^{\circ},-20.6^{\circ}$,

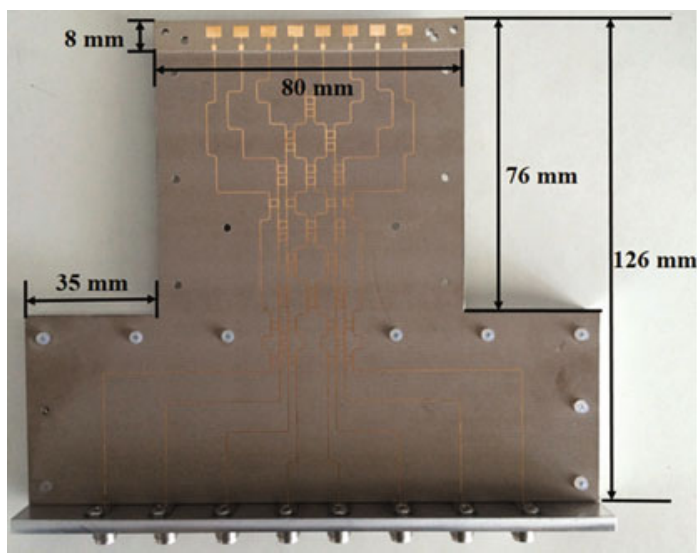

Figure 12 Photograph of designed switched-beam antenna $-22.7^{\circ},-27.9^{\circ},-14.8^{\circ},-24.2^{\circ}$, and $-20.4^{\circ}$ when the theoretical constant is $-22.5^{\circ}$, which is shown in Fig.13 and Tab.1. Owing to the different lengths of the transmission microstrip lines in the Butler matrix network, the output amplitude and the difference in phase may be imbalanced. The simulated transmission amplitudes at $25 \mathrm{GHz}$ from port 1 to the eight output ports are in a range of $-9.3 \mathrm{~dB}$ to $-10.6 \mathrm{~dB}$ when the theoretical constant is $-9 \mathrm{~dB}$, as shown in Fig.14. This indicates that the insertion loss is less than $1.6 \mathrm{~dB}$. The simulated transmission phases from port 1 to the eight output ports are shown in Fig.15.

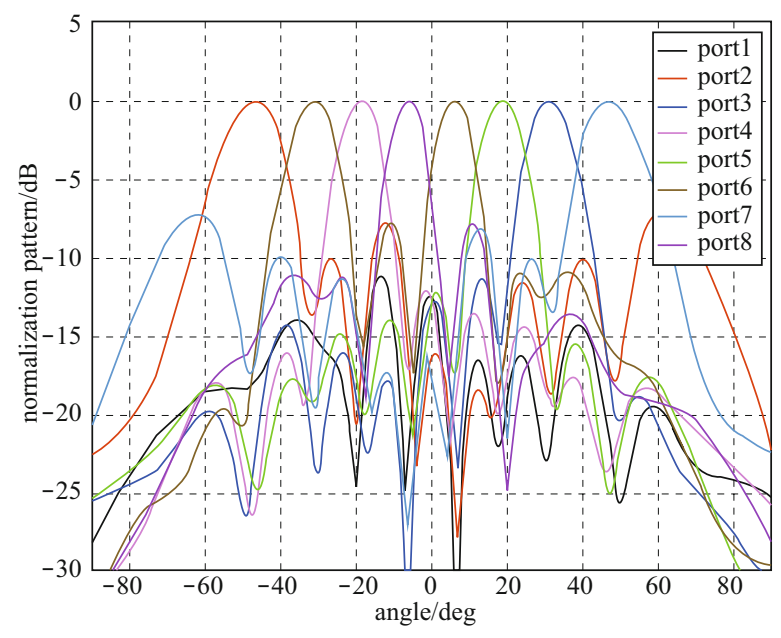

Figure 13 Simulated normalization pattern

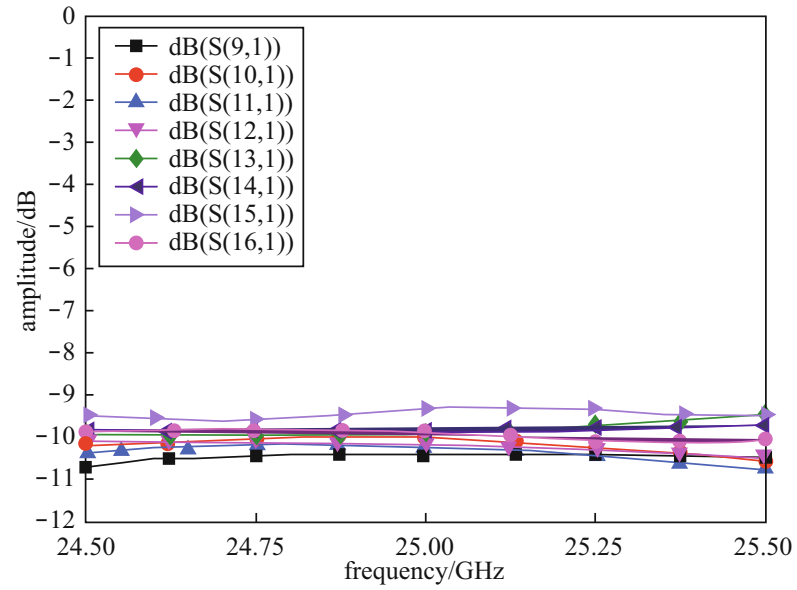

Figure 14 Simulated transmission amplitude of Butler matrix network with port 1 as input port 


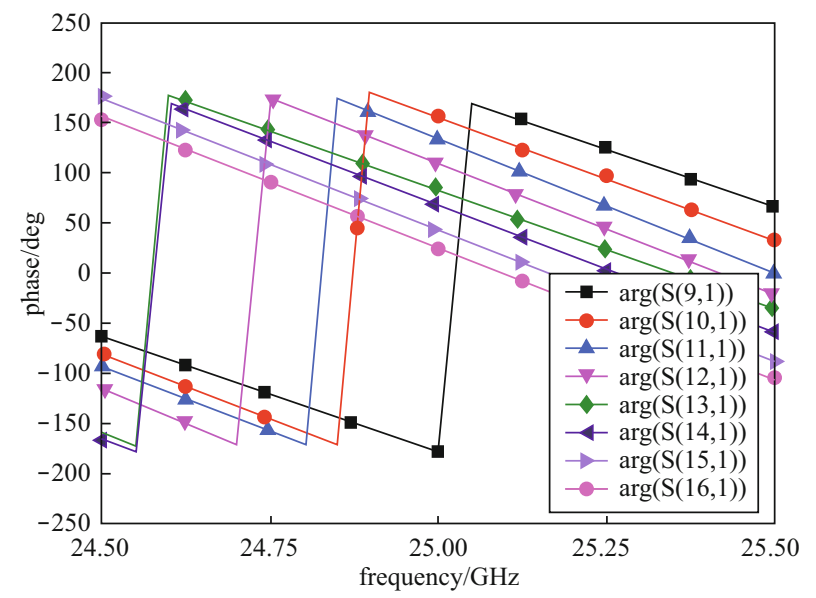

Figure 15 Simulated transmission phase of Butler matrix network with port 1 as input port

Table 1 Angles of beam center with different input ports

\begin{tabular}{ccc}
\hline input port & phase difference & angle of beam center \\
\hline 1 & $-22.5^{\circ}$ & $6^{\circ}$ \\
2 & $157.5^{\circ}$ & $-47^{\circ}$ \\
3 & $-112.5^{\circ}$ & $31^{\circ}$ \\
4 & $67.5^{\circ}$ & $-19^{\circ}$ \\
5 & $-67.5^{\circ}$ & $19^{\circ}$ \\
6 & $112.5^{\circ}$ & $-31^{\circ}$ \\
7 & $-157.5^{\circ}$ & $47^{\circ}$ \\
8 & $22.5^{\circ}$ & $-6^{\circ}$ \\
\hline
\end{tabular}

According to the results above, it is seen that the antenna array based on Butler matrix can realize beam switching.

\subsection{Beam searching and tracking}

With regard to the communication procedure between the UE and BS, first, UE establishes reliable access to the sub-3-GHz control BS and gains a reliable control connection to the network side. Then, with the help of the control BS, the mmWave BS and UE will start processing the beam sweeping and selection. Next, the communication channel can be set up owing to the available beam combination between the UE and mmWave BS. In addition, in order to support the movement of the UE, a beam-tracking algorithm is also developed.

Using the test bench, an experiment is conducted to illustrate the tracking algorithm performance. As shown in Fig.16, there are two available transmitand-receive beam direction combinations, expressed as $8 / 8$ (the 8 th beam at the transmitter side and the 8th beam at the receiver side) caused by LOS (Line Of Sight) and 5/6 (the 5th beam at the transmitter side and the 6th beam at the receiver side), which is NLOS (Non-Line Of Sight) when reflected by the reflector. As shown in Fig.17, owing to the blockage of the LOS path, the receive signal power of LOS drops abruptly at approximately $10 \mathrm{~dB}$. By using a tracking algorithm with the help of control coverage, the system hands off to the NLOS path by reselecting the beam direction combination 5/6 when the blockage occurs. This maintains the strength of the receiving signal.

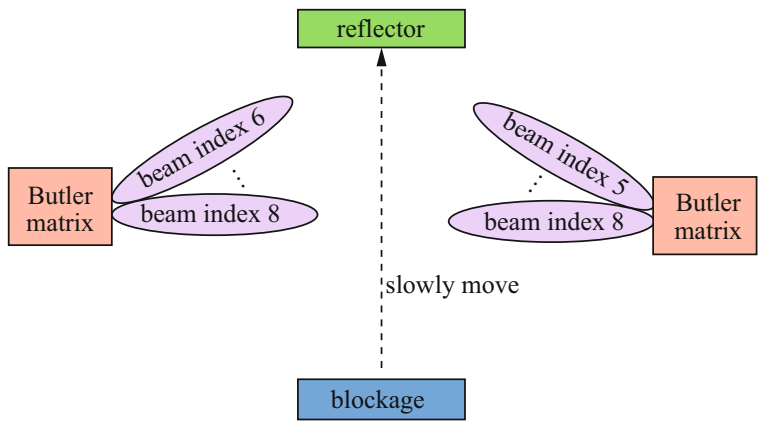

Figure 16 Test case

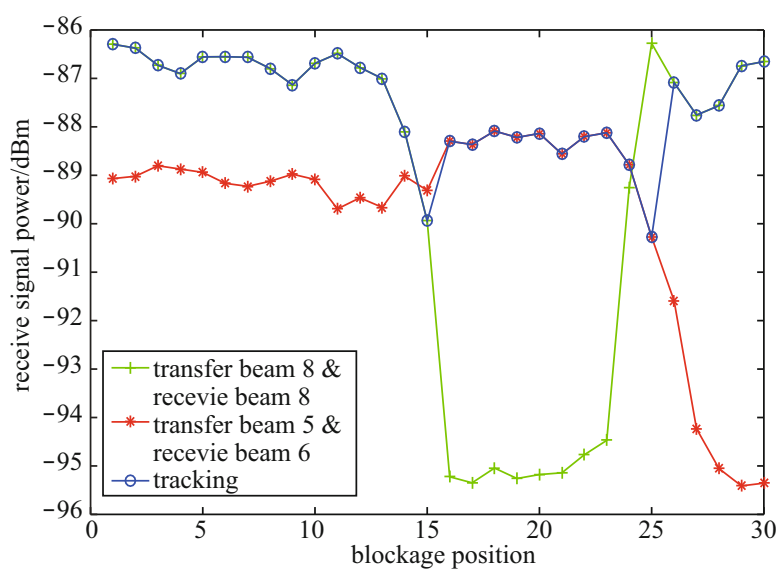

Figure 17 Tracking performance with blockage 
Therefore, it can be seen that using a hypercellular architecture in an mmWave system can overcome the problems caused by blockages.

\section{Conclusion}

In this article, we presented some new designs for mmWave systems, including network architecture and transceiver architecture. Hypercellular architecture with control/traffic separation was introduced. This architecture can reduce overhead and optimize network coverage. A Butler-matrix-based HBF architecture was proposed for the transceiver architecture. Related algorithms were proposed for the Butler-matrix-based system. Simulations were provided to verify the advantages of the Butlermatrix-based system, which can achieve an acceptable rate of performance and a better EE performance than that of a variable-phase-shifter-based system. We also presented a test bench design for the Butlermatrix-based system, which utilizes the hypercellular architecture. Experimental results were provided to validate its effectiveness.

\section{References}

[1] Cisco. Cisco visual networking index: global mobile data traffic forecast update, 2015-2020 white paper[Z]. 2016.

[2] PI Z, KHAN F. An introduction to millimeter-wave mobile broadband systems[J]. Communications magazine, IEEE, 2011, 49(6): 101-107.

[3] RANGAN S, RAPPAPORT T S, ERKIP E. Millimeter-wave cellular wireless networks: potentials and challenges[J]. Proceedings of the IEEE, 2014, 102(3): 366-385.

[4] NIU Y, LI Y, JIN D, et al. A survey of millimeter wave communications (mmWave) for $5 \mathrm{G}$ : opportunities and challenges[J]. Wireless networks, 2015, 21(8): 2657-2676.

[5] RAPPAPORT T S, SUN S, MAYZUS R, et al. Millimeter wave mobile communications for $5 \mathrm{G}$ cellular: it will work! [J]. Access, IEEE, 2013, 1: 335-349.

[6] ROH W, SEOL J Y, PARK J, et al. Millimeter-wave beamforming as an enabling technology for $5 \mathrm{G}$ cellular communications: theoretical feasibility and prototype results[J]. Communications magazine, IEEE, 2014, 52(2): 106-113.

[7] CUDAK M, KOVARIK T, THOMAS T A, et al. Experimental mmwave 5G cellular system[C]//Globecom Workshops (GC Wkshps), IEEE, 2014: 377-381.

[8] SINGH S, ZILIOTTO F, MADHOW U, et al. Blockage and directivity in $60 \mathrm{GHz}$ wireless personal area networks: from cross-layer model to multihop MAC design[J]. Selected areas in communications, IEEE journal on, 2009, 27(8): 1400-1413.

[9] NIU Z, ZHOU S, ZHOU S, et al. Energy efficiency and resource optimized hyper-cellular mobile communication system architecture and its technical challenges[J]. SCIENTIA SINICA informationis, 2012, 42(10): 1191-1203.

[10] EL AYACH O, RAJAGOPAL S, ABU-SURRA S, et al. Spatially sparse precoding in millimeter wave MIMO systems[J]. Wireless communications, IEEE transactions on, 2014, 13(3): 1499-1513.

[11] XU X, HE G, ZHANG S, et al. On functionality separation for green mobile networks: concept study over LTE[J]. Communications magazine, IEEE, 2013, 51(5): 82-90.

[12] RANGAN S, RAPPAPORT T, ERKIP E, et al. Energy efficient methods for millimeter wave picocellular systems[J]. Communications, 2013.

[13] MENDEZ-RIAL R, RUSU C, GONZALEZ-PRELCIC N, et al. Hybrid MIMO architectures for millimeter wave communications: phase shifters or switches[J]. Mathematics, 2016, 4: 247-267.

[14] AL-JOUMAYLY M A, BEHDAD N. Wideband planar microwave lenses using sub-wavelength spatial phase shifters[J]. Antennas and propagation, IEEE transactions on, 2011, 59(12): 4542-4552.

[15] CHIN T Y, CHANG S F, WU J C, et al. A 25-GHz compact lowpower phased-array receiver with continuous beam steering in CMOS technology[J]. Solid-state circuits, IEEE journal of, 2010, 45(11): 2273-2282.

[16] TSENG C H, CHEN C J, CHU T H. A low-cost 60-GHz switchedbeam patch antenna array with Butler matrix network[J]. Antennas and wireless propagation letters, IEEE, 2008, 7: 432-435.

[17] RUSCH C, KARCHER C, BEER S, et al. Planar beam switched antenna with Butler matrix for $60 \mathrm{GHz}$ WPAN[C]//Antennas and Propagation (EUCAP), 2012 6th European Conference on. IEEE, 2012: 2794-2797.

[18] PATTERSON C E, KHAN W T, PONCHAK G E, et al. A 60-GHz active receiving switched-beam antenna array with integrated butler matrix and GaAs amplifiers[J]. Microwave theory and techniques, IEEE transactions on, 2012, 60(11): 3599-3607.

[19] MADANY Y M, ELKAMCHOUCHI H M, SALAMA A A. Design and analysis of optimum miniaturized conformal smart antenna system using $1 \times 8$ switched Butler Matrix for wireless applications[C]//Microwave and Millimeter Wave Technology (ICMMT), 2012 International Conference on. IEEE, 2012, 1: 1-4.

[20] YUJIAN L, KWAI-MAN L. A multi-beam end-fire magneto-electric dipole antenna array for millimeter-wave applications[J]. Antennas \& propagation, IEEE transactions on, 2016, 64(7): 2894-2904.

[21] ANTONIO R, GIOVANNI C. Microwave wireless communications from transistor to system level[M]. Academic Press, Amsterdam, Netherlands, 2016. 266, 280.

[22] STANKOVIC V, HAARDT M. Generalized design of multi-user MIMO precoding matrices[J]. Wireless communications, IEEE 
transactions on, 2008, 7(3): 953-961.

[23] LI J, XIAO L, XU X, et al. Energy-efficient Butler-matrix-based hybrid beamforming for multiuser mmWave MIMO system[J]. IEEE communications letters, 2016: 1-1.

[24] GAO X, DAI L, HAN S, et al. Energy-efficient hybrid analog and digital precoding for mmWave MIMO systems with large antenna arrays[J]. arXiv preprint arXiv:1507.04592, 2015.
[25] FORENZA A, LOVE D J, HEATH JR R W. Simplified spatial correlation models for clustered MIMO channels with different array configurations[J]. Vehicular technology, IEEE transactions on, 2007, 56(4): 1924-1934

[26] HAN S, CHIH-LIN I, XU Z, et al. Large-scale antenna systems with hybrid analog and digital beamforming for millimeter wave $5 \mathrm{G}[\mathrm{J}]$. Communications magazine, IEEE, 2015, 53(1): 186-194.

\section{About the authors}

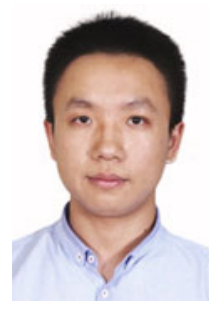

tsinghua.edu.cn)

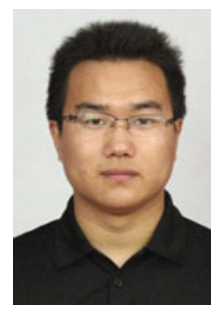

HUANG Fei was born in Anhui, China. He received the B.S. degree in electronic engineering from Tsinghua University, Beijing, China, in 2015, and is currently working toward the M.S. degree in electronic engineering at Tsinghua University. His research interests include millimeter-wave transmitting systems and high-efficiency RF PA design. (Email: huang-f15@mails.tsinghua.edu.cn)

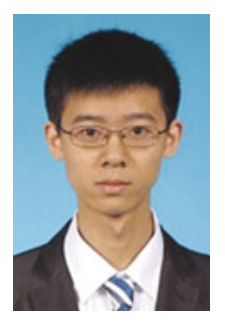

ZHOU Runyun was born in 1991. He received the B.E. degree in electronic engineering from Tsinghua University, Beijing, China. He is now a M.S. candidate of the Wireless and Mobile Communication Technology R\&D Center of Tsinghua University. His research interests include SDR and the network architecture of future wireless communication. (Email: zrybrent@126.com)

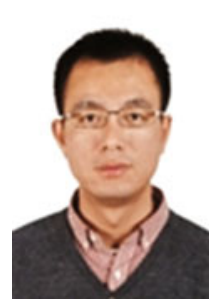

CHEN Wenhua received the B.S. degree in microwave engineering from the University of Electronic Science and Technology of China (UESTC), Chengdu, in 2001, and the Ph.D. degree in electronic engineering from Tsinghua University, Beijing, China, in 2006. From 2010 to 2011, he was a Postdoctoral Fellow with the Intelligent RF Radio Laboratory (iRadio Lab),
University of Calgary. He is currently an Associate Professor with the Department of Electronic Engineering, Tsinghua University, Beijing, China. His main research interests include power-efficiency enhancement for wireless transmitters, PA predistortion, and smart antennas. He has authored or coauthored over 120 journal and conference papers. His main research interests include RF power amplifier design and linearization digital assisted RF circuit technique, energy-efficient millimeter wave transmitter. Dr. Chen is as an associate editor for the International Journal of Microwave and Wireless Technology. He was the recipient of the 2015 Outstanding Youth Science Foundation of NSFC, the 2014 URSI Young Scientist Award, the Student Paper Award of the 2010 Asia-Pacific Microwave Conference (APMC). (Email: chenwh@tsinghua.edu.cn)

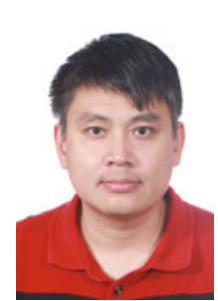

TIAN Zhigang was born in ShanXi, China. He received the B.E. degree and Master degree in electronic engineering from Beijing Jiaotong University, and received Ph.D. degree in electronic engineering from Beijing University of Post and Telecommuication. His research interests include 5G network architecture and 5G MAC algorithm and protocol. (Email: zgtian@tsinghua.edu.cn)

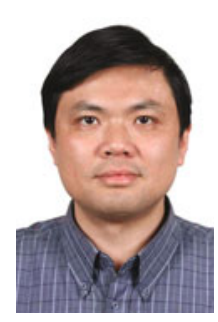

ZHOU Shidong [corresponding author] received BS and MS degree from Southeast University, Nanjing, in 1991 and 1994 respectively, and Received Ph.D. degree from Tsinghua University in 1998. He is now working as a professor with Department of Electronic Engineering, Tsinghua University. He was engaged in several major national projects on $3 \mathrm{G}$ and $4 \mathrm{G}$ mobile communication technique research and development. His current research interest includes: mobile communication system architectures, advanced transmission technique, wireless channel sounding and modeling, radio resource management, high energy efficient wireless networks. (Email: zhousd@tsinghua.edu.cn) 